\title{
Photon and photon+jet production measurements with the ATLAS detector
}

\author{
Martin Bessner* on behalf of the ATLAS collaboration \\ DESY
}

E-mail: martin.bessner@cern.ch

\begin{abstract}
Isolated prompt photons provide a direct probe of short-distance physics, complementary to that provided by measurements of jets or vector-bosons and are sensitive to the gluon density of the proton. The inclusive prompt photon cross sections have been measured by the ATLAS collaboration at $7 \mathrm{TeV} p p$ collision centre-of-mass energies, over a wide range of transverse momenta. The diphoton and photon+jet system cross sections have also been measured as a function of several kinematic variables. These experimental results are reported in different fiducial regions covering a wide acceptance and are compared to next-to-leading order QCD calculations with different models of the parton content of the proton. Early LHC Run-2 results are included.
\end{abstract}

The European Physical Society Conference on High Energy Physics

22-29 July 2015

Vienna, Austria

\footnotetext{
* Speaker.
} 


\section{Introduction}

Photons are an important tool to study proton-proton collisions in colliders. They can be produced in hard interactions, but unlike quarks and gluons they do not participate in hadronization afterwards. Therefore, they allow to probe perturbative quantum chromodynamics in an experimentally clean way. Studies of photon production also help to constrain proton parton density functions (PDFs) and allow to improve the accuracy of theoretical predictions.

These proceedings present measurements of production cross sections for photons and photons plus jets with the ATLAS detector [1] at proton-proton collision energies of $\sqrt{s}=7 \mathrm{TeV}$ and $\sqrt{s}=13 \mathrm{TeV}$.

\section{Photon identification in the ATLAS detector}

Photon identification in the ATLAS detector mainly relies on the electromagnetic calorimeter, a liquid argon calorimeter that surrounds the tracking system. It covers the pseudorapidity range of $|\eta|<3.2$ with a combination of barrel and endcap calorimeters.

Photons deposit most of their energy in the electromagnetic calorimeter. Energy deposits in the hadronic calorimeter can be used to veto hadrons. Photons are reconstructed as unconverted (no track in the tracking system) or converted (one or two tracks) photon candidates. Events are triggered based on one or two photon candidates above transverse energy thresholds.

In order to suppress jet background, 9 different discriminating variables describing the shape of the shower in the calorimeter are used. The identification is done separately for unconverted and converted photon candidates. An additional requirement of photon isolation further improves the background rejection: the sum of transverse energy of all positive-energy calorimeter clusters in a cone of $\Delta R=0.4$ around the photon is required to be below some specific threshold. This threshold varies between 3 to $7 \mathrm{GeV}$, depending on the analysis. Combined, the background rejection is at the level of 5000 with an efficiency of around $90 \%$ for high-energetic photons [2].

Photon identification requires the fine granularity of the first calorimeter layer. This restricts the studies to the barrel calorimeter region of $|\eta|<1.37$ and the endcap calorimeter region of $1.52<|\eta|<2.37$.

\section{Inclusive photon production}

The cross section of isolated prompt photons in $\sqrt{s}=7 \mathrm{TeV}$ collisions has been studied based on an integrated luminosity of $4.6 \mathrm{fb}^{-1}$ [3]. The measurement extends previous results up to $E_{\mathrm{T}}^{\gamma}=1 \mathrm{TeV}$. The dominant production process is $q g \rightarrow q \gamma$, this allows to measure the gluon content of the proton, and it is used to constrain the gluon PDF.

The distributions of prompt photons are compared to theoretical predictions as a function of transverse energy $E_{\mathrm{T}}^{\gamma}$ and pseudorapidity $\left|\eta^{\gamma}\right|$, as shown in figure 1. The theoretical predictions underestimate the cross section over the whole pseudorapidity range, mainly due to photons with $E_{\mathrm{T}}^{\gamma}<200 \mathrm{GeV}$. For high $E_{\mathrm{T}}^{\gamma}$, predictions are slightly above the measured cross sections. Both deviations are within the uncertainties of the next-to-leading order (NLO) predictions. As the theoretical uncertainties due to PDFs are significant for high $E_{\mathrm{T}}^{\gamma}$, the measurements provide constraints on the proton PDFs. 

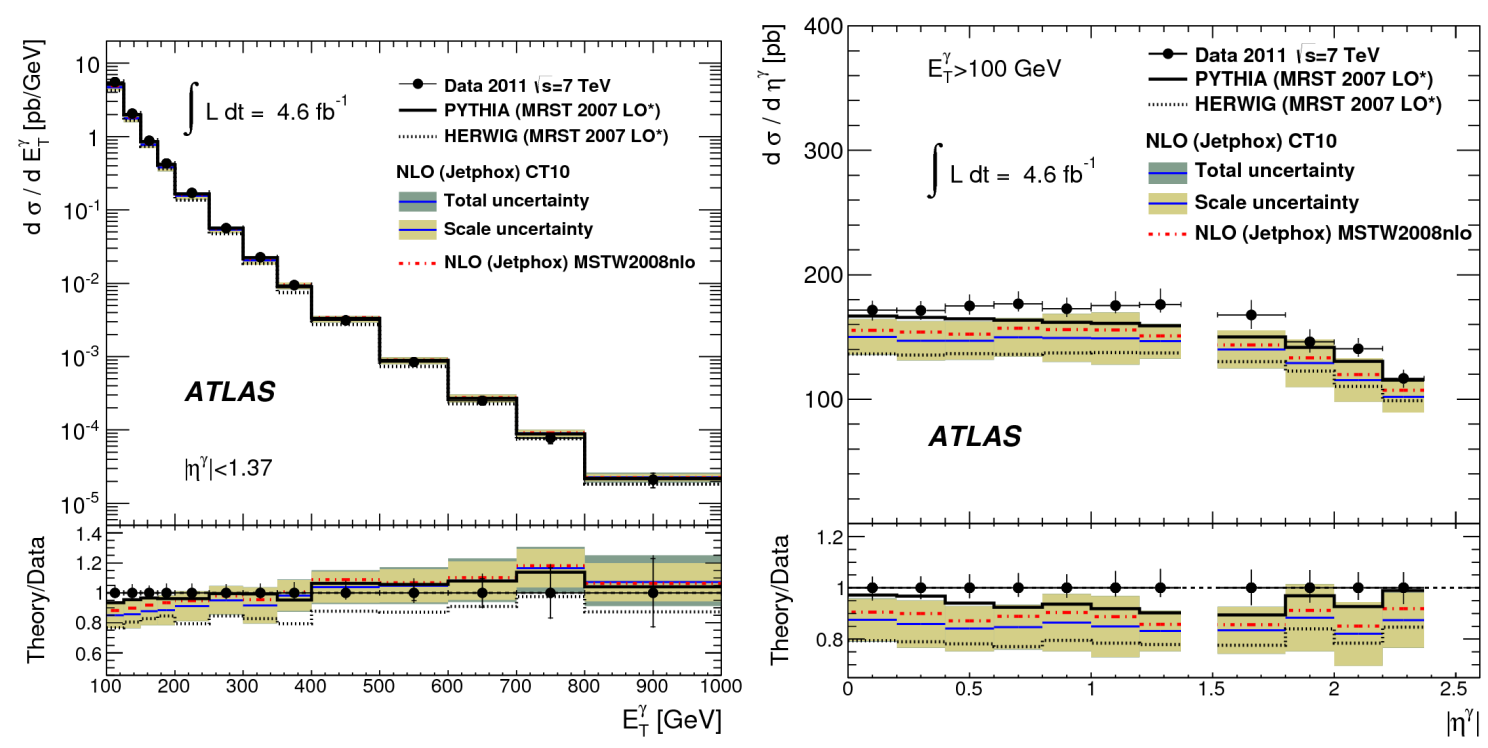

Figure 1: Differential cross section of prompt photons as a function of the transverse energy $E_{\mathrm{T}}^{\gamma}$ and the pseudorapidity $\left|\eta^{\gamma}\right|$ [3]. The theoretical predictions underestimate the production over the whole pseudorapidity range, mainly due to photons with $E_{\mathrm{T}}^{\gamma}<200 \mathrm{GeV}$.

\section{Photon and jet production}

In events with a photon, the additional requirement of a jet allows the measurement of more observables. Based on an integrated luminosity of $37 \mathrm{pb}^{-1}$, the photon plus jet production cross section has been studied as a function of the photon transverse energy, the jet transverse momentum, the jet rapidity, the difference between azimuthal angle of photon and jet, the invariant mass of the photon-jet system and the scattering angle $\theta^{\gamma j}$ in the photon-jet center-of-mass frame [4].

The two main production modes are direct production $(q g \rightarrow \gamma q)$, mediated by a quark, and fragmentation ( $q q \rightarrow q q$ where one quark emits a photon), mediated by a gluon. The different spin of the exchange particle leads to different spectra. The most prominent difference is visible in the distribution of $\cos \theta^{\gamma j}=\tanh \left(\frac{\Delta y}{2}\right)$, where $\Delta y$ is the difference between photon and (leading) jet rapidity.

The detector acceptance and the selection based on transverse energy lead to a larger acceptance for small $\cos \theta^{\gamma j}$. In order to avoid a bias on the measured shape, selection criteria on the sum of photon pseudorapidity and jet rapidity and on the invariant mass of the photon-jet system are added. The comparison to theoretical predictions is shown in figure 2.

A good description by JETPHOX NLO predictions can be observed in all variables studied, both within the full detector acceptance and with the smaller unbiased phase space for $\cos \theta^{\gamma j}$.

\section{Diphoton production}

The production of pairs of isolated photons has been studied based on an integrated luminosity of $4.9 \mathrm{fb}^{-1}$ [5]. The differential cross sections as a function of the diphoton invariant mass, transverse momentum $p_{\mathrm{T}, \gamma \gamma}$, azimuthal separation $\Delta \phi_{\gamma \gamma}$ and cosine of the polar angle of the leading photon (largest transverse energy) in the Collins-Soper diphoton rest frame are measured. 

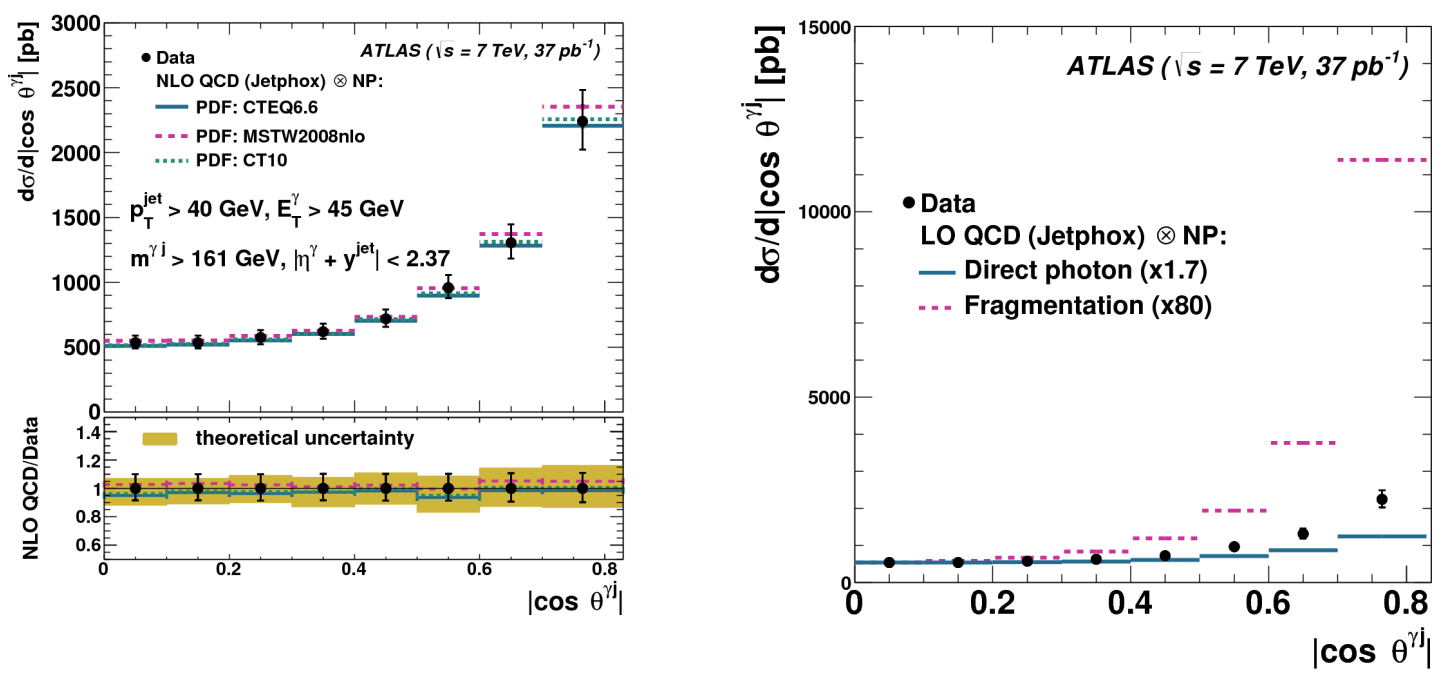

Figure 2: Left: cross section of photon and jet production as a function of $\cos \theta^{\gamma j}$ which depends on the difference between the azimuthal angles of photon and jet. The measurement is compared to NLO predictions from JETPHOX using three different PDF sets. Right: A comparison of the measured shape with the expectations from pure fragmentation (dashed line) and pure direct production (solid line). The experimental result is closer to the shape of direct production, showing the dominance of this production mode [4].
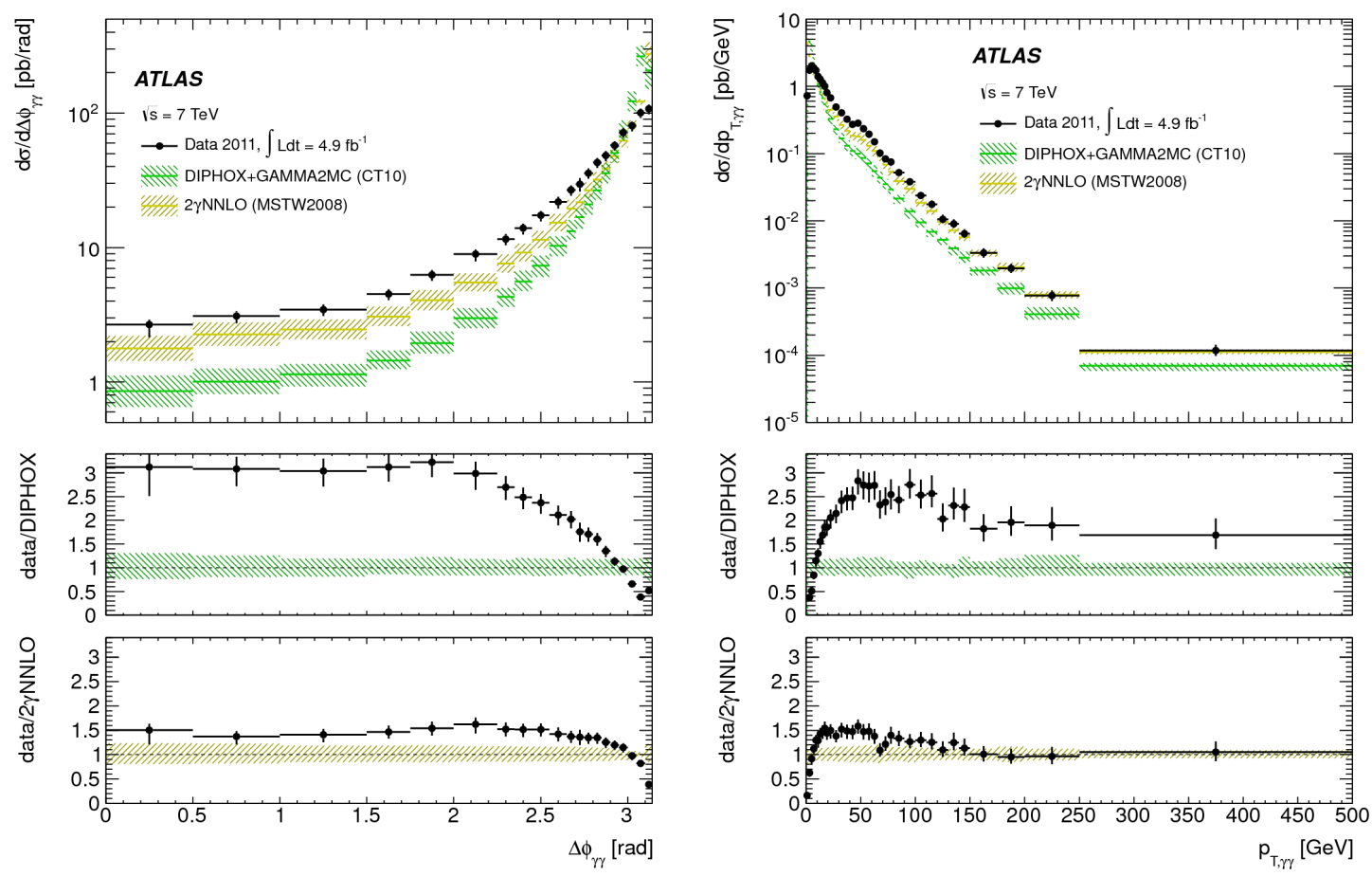

Figure 3: Azimuthal separation and transverse momentum of the diphoton system. Deviations from nextto-next-to-leading order (NNLO) predictions are visible [5]. 
Understanding the invariant mass distribution is relevant for searches for resonances, transverse momentum and azimuthal separation are used to study perturbative QCD, the cosine of the polar angle is sensitive to the spin of possible diphoton resonances, similar to the photon and jet production measurement described above.

The measured spectra are compared to various predictions up to next-to-next-to-leading order (NNLO). The effects of missing higher orders are visible in LO and NLO predictions, while NNLO predictions provide a reasonable agreement with measurements. Deviations are visible in the $\Delta \phi_{\gamma \gamma}$ spectrum and for low $p_{\mathrm{T}, \gamma \gamma}$ (see figure 3), those are expected for calculations at fixed order without fragmentation component.

\section{6. $13 \mathrm{TeV}$ results}
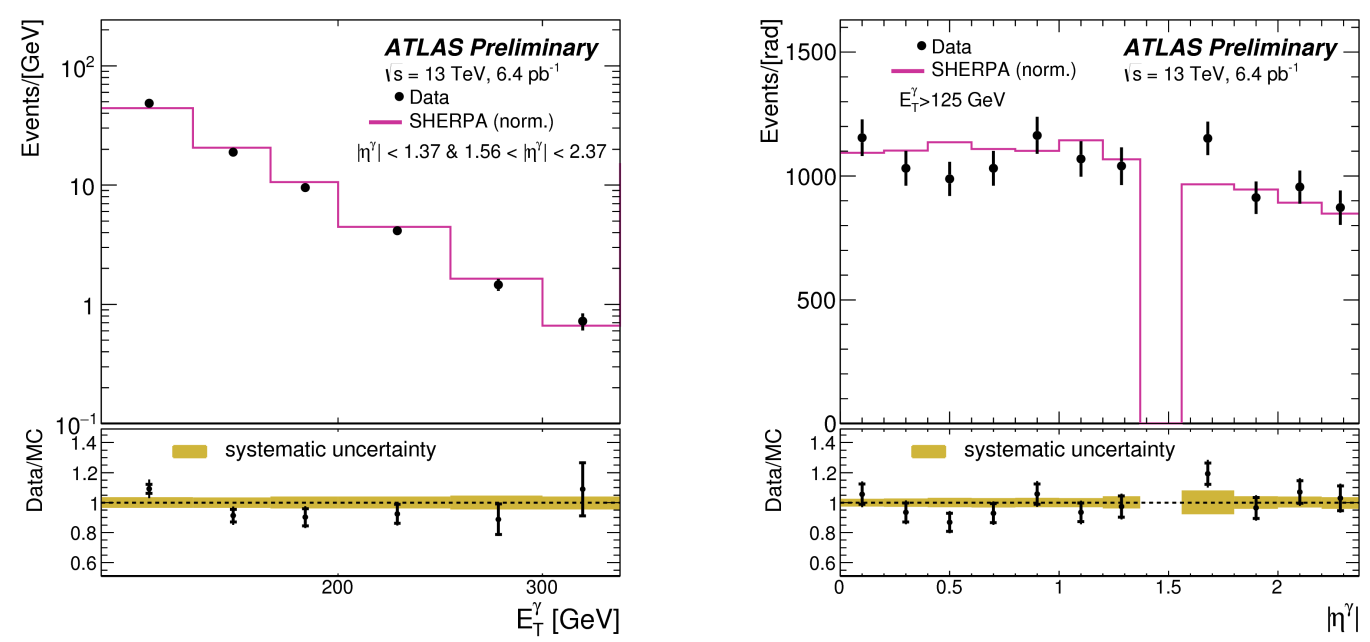

Figure 4: Inclusive photon spectra as a function of transverse energy $E_{\mathrm{T}}$ and pseudorapidity $\eta$ in $\sqrt{s}=13 \mathrm{TeV}$ collisions [6].

Photon production in $\sqrt{s}=13 \mathrm{TeV}$ collisions has been studied, based on a dataset corresponding to an integrated luminosity of $6.4 \mathrm{pb}^{-1}$.

The inclusive photon production up to $E_{\mathrm{T}}=350 \mathrm{GeV}$ is shown in figure 4, the shapes of the $E_{\mathrm{T}}$ and $\eta$ spectra show a good agreement with predictions based on SHERPA using leading-order matrix elements with up to three additional partons and initial- and final-state parton showers [6].

Spectra of diphoton candidates for the invariant mass and transverse momentum of the diphoton system [7] are shown in figure 5.

\section{Conclusions}

A selection of ATLAS results on photon and photon plus jet production has been shown. In general, theoretical predictions match the experimental results reasonably well, but some deviations 

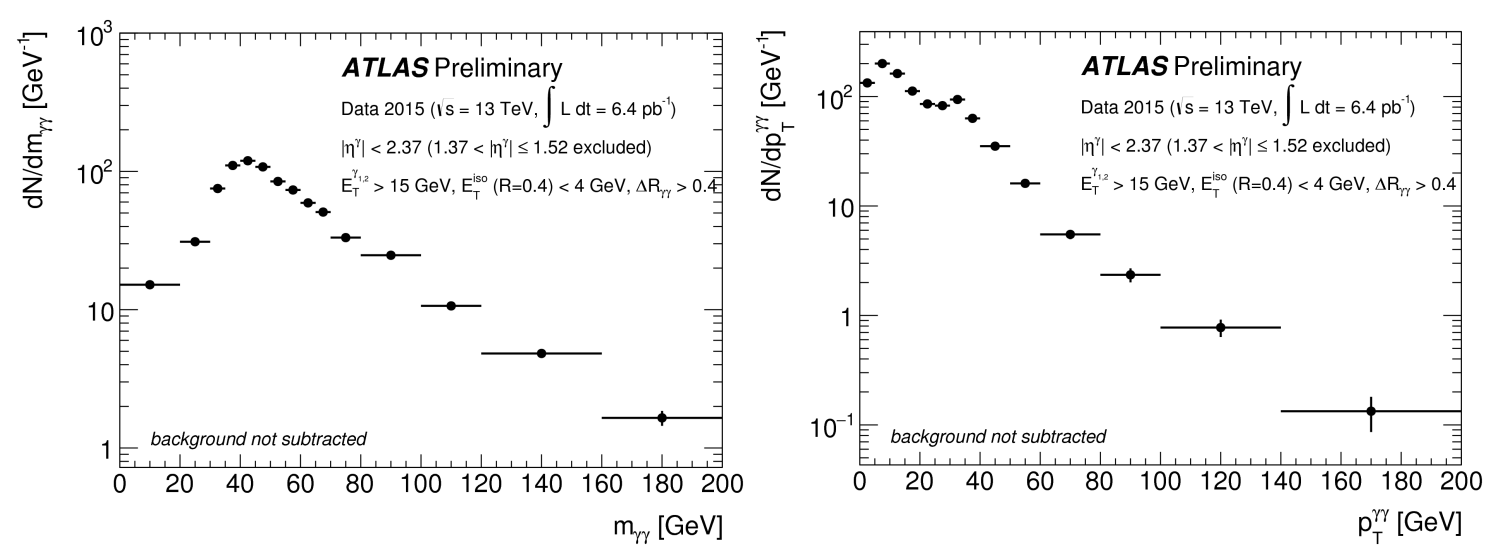

Figure 5: Spectra of diphoton candidates as a function of diphoton invariant mass $m_{\gamma \gamma}$ and transverse momentum $p_{\mathrm{T}}^{\gamma \gamma}$ in $\sqrt{s}=13 \mathrm{TeV}$ collisions [7].

are visible. The measurements help to constrain the proton PDFs, especially the gluon contribution. With increasing experimental precision, NLO and NNLO calculations are important.

The studies are extended to a higher collision energy of $\sqrt{s}=13 \mathrm{TeV}$ from Run 2 .

\section{References}

[1] ATLAS Collaboration, The ATLAS experiment at the CERN Large Hadron Collider, JINST 3 S08003 (2008).

[2] ATLAS Collaboration, Measurements of the photon identification efficiency with the ATLAS detector using $4.9 \mathrm{fb}^{-1}$ of pp collision data collected in 2011, ATLAS-CONF-2012-123, http://cdsweb.cern.ch/record/1473426

[3] ATLAS Collaboration, Measurement of the inclusive isolated prompt photon cross section in pp collisions at $\sqrt{s}=7 \mathrm{TeV}$ with the ATLAS detector using $4.6 \mathrm{fb}^{-1}$, Phys. Rev. D 89, 052004 (2014) (arXiv:1311.1440 [hep-ex])

[4] ATLAS Collaboration, Dynamics of isolated-photon and jet production in pp collisions at $\sqrt{s}=7 \mathrm{TeV}$ with the ATLAS detector, Nucl. Phys, B 875 (2013) 483-535 (arXiv:1307.6795 [hep-ex])

[5] ATLAS Collaboration, Measurement of isolated-photon pair production in pp collisions at $\sqrt{s}=7$ TeV with the ATLAS detector, JHEP01(2013)086 (arXiv:1211.1913 [hep-ex])

[6] ATLAS Collaboration, Study of inclusive isolated-photon production in pp collisions at $\sqrt{s}=13 \mathrm{TeV}$ with the ATLAS detector, ATL-PHYS-PUB-2015-016 http://cdsweb.cern.ch/record/2037667

[7] ATLAS Collaboration, Inclusive spectra of isolated di-photon candidates using $6.4 \mathrm{pb}^{-1}$ of pp collisions at $\sqrt{s}=13 \mathrm{TeV}$, ATL-PHYS-PUB-2015-020, http://cdsweb.cern.ch/record/2037687 